Roland Glowinski · Jiwen He • Alexei

Lozinski* · Jacques Rappaz • Joël Wagner

\title{
Finite element approximation of multi-scale elliptic problems using patches of elements
}

Received: 6 April 2004 / Published online: 18 July 2005

(c) Springer-Verlag 2005

\begin{abstract}
In this paper we present a method for the numerical solution of elliptic problems with multi-scale data using multiple levels of not necessarily nested grids. The method consists in calculating successive corrections to the solution in patches whose discretizations are not necessarily conforming. This paper provides proofs of the results published earlier (see C. R. Acad. Sci. Paris, Ser. I 337 (2003) 679-684), gives a generalization of the latter to more than two domains and contains extensive numerical illustrations. New results including the spectral analysis of the iteration operator and a numerical method to evaluate the constant of the strengthened Cauchy-Buniakowski-Schwarz inequality are presented.
\end{abstract}

Mathematics Subject Classifications (1991) 65N55 - 65N30 - 65N12

\section{Introduction}

The numerical approximation of the solution of elliptic partial differential equations in domains such that in certain regions a "better" precision on the solution is needed leads to many interesting issues. Efficient approaches include adaptive mesh refinement techniques, domain decomposition methods and multigrid methods. The objective of this paper is to present a method to solve numerically elliptic problems with multi-scale data using multiple levels of not necessarily nested grids.

*Supported by CTI Project 6437.1 IWS-IW.

Roland Glowinski · Jiwen He

Department of Mathematics, University of Houston, 4800 Calhoun Road, Houston,

Texas 77204-3008, USA

E-mail: \{roland,jiwenhe\}@ math.uh.edu

Alexei Lozinski · Jacques Rappaz · Joël Wagner $(\varangle)$

Section of Mathematics, Swiss Federal Institute of Technology, 1015 Lausanne, Switzerland E-mail: \{alexei.lozinski,jacques.rappaz,joel.wagner\}@epfl.ch 
A motivation for developing such a method can be found, for example, in air quality management. Pollution emission sources, and in particular point source plumes, give rise to models needing careful examination of the space-scale. Getting an accurate simulation on large scales is linked to a simulation in subregions around the pollution sources using finer grids. Such a method can be applied straightforwardly to boundary layer problems through the use of patches in critical regions, or in the coupling of problems with nonconforming grids for example.

We solve the problem on a domain $\Omega$ and consider therein patches $\Lambda_{1}, \Lambda_{2}, \ldots$ wherein we would like to obtain more accuracy (see Fig. 1). Thus we calculate successively corrections to the solution in the patches. The discretizations of the latter are not necessarily conforming. The method is a domain decomposition method with complete overlapping. It resembles the Fast Adaptive Composite grid (FAC) method (see, e.g., [31]) or possibly a hierarchical method (see [16] for example). However it is of much more flexible use in comparison to the latter.

In Section 2 we first introduce the correction algorithm (Algorithm 1) in the case of two scales, i.e. with the domain $\Omega$ and one only patch $\Lambda$. We give an a priori error estimate for the $h$-convergence in Proposition 1 . The convergence properties of the two-scale algorithm are stated in Proposition 2 through the iteration operator (10).

In Section 3 we prove some convergence results in an abstract setting. In the first paragraph we analyze some properties of vector spaces. Next we introduce an operator (22) that is to be identified with the iteration operator (10) of Algorithm 1. In Proposition 5 we recall the upper bound of its norm presented in [22]. A spectral analysis of the operator yields an exact formula for its spectral radius and norm given in Proposition 6.

In Section 4 we discuss the constant $\gamma$ of the strengthened Cauchy-Buniakowski-Schwarz (C.B.S.) inequality which appears in our convergence analysis. In the case of a patch $\Lambda$ included in only one triangle of the coarse triangulation of $\Omega$ we give a bound for $\gamma$ in the case of a scalar product corresponding to the bilinear form of an elliptic operator. We also develop some upper bounds in particular cases (see Fig. 2) and give a new method to estimate it numerically.

In Section 5 we generalize Algorithm 1 and some results obtained in Section 3 for two spaces to multiple spaces. We establish a generalization of Proposition 5 in Proposition 9 which is used to prove the convergence of the multi-scale algorithm in Proposition 8.

Section 6 gives some numerical results. In $\$ 6.1$ we present numerical estimates of $\gamma$ for some cases treated in Section 4 (Table 2) and new grid constellations

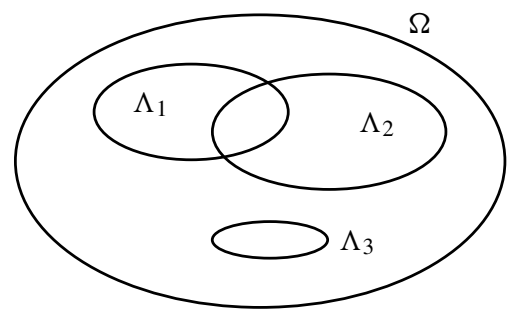

Fig. 1 Domain $\Omega$ with patches 
(Table 3) that we use in $\$ 6.2$ to illustrate the convergence behavior of the algorithm. In $\$ 6.3$ we illustrate the a priori estimate for the convergence in the mesh size (Fig. 6).

\section{Two-scale algorithm}

Let $\Omega \subset \mathbb{R}^{2}$ be an open polygonal domain and consider a bilinear, symmetric, continuous and coercive form

$$
a: H_{0}^{1}(\Omega) \times H_{0}^{1}(\Omega) \rightarrow \mathbb{R} .
$$

The usual $H^{1}(\Omega)$-norm is equivalent to the $a$-norm defined by $\|v\|=a(v, v)^{\frac{1}{2}}$, $\forall v \in H_{0}^{1}(\Omega)$. If $f \in H^{-1}(\Omega)$, due to Riesz' representation Theorem there exists a unique $u \in H_{0}^{1}(\Omega)$ such that

$$
a(u, v)=\langle f \mid v\rangle, \quad \forall v \in H_{0}^{1}(\Omega),
$$

where $\langle\cdot \mid \cdot\rangle$ denotes the duality $H^{-1}(\Omega)-H_{0}^{1}(\Omega)$. Let us point out that $(1)$ is the weak formulation of a problem of type $\mathcal{L}(u)=f$ in $\Omega, u=0$ on the boundary $\partial \Omega$ of $\Omega$, where $\mathcal{L}(\cdot)$ is a second order, linear, symmetric, strongly elliptic operator. An approximation of $u$ by the finite element method of order $r$ consists in introducing a regular triangulation $\mathcal{T}_{H}$ of $\bar{\Omega}$ (see [20], Sect. 17), defining

$$
\begin{aligned}
V_{H}=\left\{g: \bar{\Omega} \rightarrow \mathbb{R} \text { continuous such that }\left.g\right|_{K} \in \mathbb{P}_{r}(K), \forall K \in \mathcal{T}_{H}\right. \\
\text { and } g=0 \text { on } \partial \Omega\},
\end{aligned}
$$

where $\mathbb{P}_{r}(K)$ is the space of polynomials of degree $\leq r$ on triangle $K \in \mathcal{T}_{H}$, and calculating $u_{H} \in V_{H}$ satisfying

$$
a\left(u_{H}, v\right)=\langle f \mid v\rangle, \quad \forall v \in V_{H} .
$$

Consider now $\Lambda \subset \Omega$ another open polygonal domain wherein we would like to obtain a better precision on the solution $u$ than the one given by $u_{H}$. Take note that $\bar{\Lambda}$ is not necessarily the union of several triangles $K$ of $\mathcal{T}_{H}$. Besides $\Lambda$ can be determined in practice by an a priori knowledge or an a posteriori error estimator, for example. Let $\mathcal{T}_{h}$ be a regular triangulation of $\bar{\Lambda}$ and consider

$$
\begin{aligned}
& V_{h}=\left\{g: \bar{\Omega} \rightarrow \mathbb{R} \text { continuous such that }\left.g\right|_{K} \in \mathbb{P}_{S}(K), \forall K \in \mathcal{T}_{h}\right. \\
& \text { and } g=0 \text { on } \bar{\Omega} \backslash \Lambda\} .
\end{aligned}
$$

We call $H=\max _{K \in \mathcal{T}_{H}} \operatorname{diam}(K)$ and $h=\max _{K \in \mathcal{T}_{h}} \operatorname{diam}(K)$. Setting $V_{H h}=$ $V_{H}+V_{h}$ we search as approximation for $u$ the function $u_{H h} \in V_{H h}$ satisfying

$$
a\left(u_{H h}, v\right)=\langle f \mid v\rangle, \quad \forall v \in V_{H h} .
$$

Let us observe that in practice, it is not possible to determine a finite element basis of $V_{H h}$ since, in principle, $V_{H} \cap V_{h}$ is not necessarily reduced to zero. Before to show how to compute $u_{H h}$, we establish the following a priori estimate: 
Proposition 1 Let $q=\max (r, s)+1$ and suppose that the solution $u$ of $(1)$ is in $H^{q}(\Omega)$. Then the approximation $u_{H h}$ to $u$ satisfies the a priori error estimate

$$
\left\|u-u_{H h}\right\| \leq C\left(H^{r}\|u\|_{H^{q}(\Omega \backslash \bar{\Lambda})}+h^{s}\|u\|_{H^{q}(\Lambda)}\right),
$$

where $C$ is a constant independent of $H$ and $h$.

Proof The boundary $\partial \Lambda$ being locally Lipschitz, due to the Stein Extension Theorem (see Adams and Fournier [3], Thm. 5.24), there exists a bounded extension operator $E: H^{q}(\Omega \backslash \bar{\Lambda}) \rightarrow H^{q}(\Omega)$, i.e. $\left.E v\right|_{\Omega \backslash \bar{\Lambda}}=\left.v\right|_{\Omega \backslash \bar{\Lambda}}, \forall v \in H^{q}(\Omega \backslash \bar{\Lambda})$. Let $u$ be the solution of (1). We define $\tilde{u}$ the extension of $\left.u\right|_{\Omega \backslash \Lambda}$ to $\Omega$ such that $\tilde{u}=E u$ if $\|E u\|_{H^{q}(\Lambda)} \leq\|u\|_{H^{q}(\Lambda)}$ and $\tilde{u}=u$ otherwise. We have that $\tilde{u}=u$ in $\Omega \backslash \bar{\Lambda}$,

$$
\|\tilde{u}\|_{H^{q}(\Omega)} \leq C\|u\|_{H^{q}(\Omega \backslash \bar{\Lambda})},
$$

where here, like in the sequel, $C$ denotes a generic constant, and

$$
\|\tilde{u}\|_{H^{q}(\Lambda)} \leq\|u\|_{H^{q}(\Lambda)} .
$$

Note that $u-\tilde{u} \in H_{0}^{q}(\Lambda)$. Let $r_{H}$ and $r_{h}$ be the standard interpolants to the spaces $V_{H}$ and $V_{h}$ respectively. We introduce $\tilde{u}_{H}=r_{H} \tilde{u}$ and $\tilde{u}_{h}=r_{h}(u-\tilde{u})$. Define $\tilde{u}_{H h}=\tilde{u}_{H}+\tilde{u}_{h}$ and $v_{H h}=u_{H h}-\tilde{u}_{H h}$. By the definitions of $u$ and $u_{H h}$ we have $a\left(u, v_{H h}\right)=a\left(u_{H h}, v_{H h}\right)$. This and the previous definitions lead to the equality $a\left(v_{H h}, v_{H h}\right)=a\left(u-\tilde{u}_{H h}, v_{H h}\right)$, from which we derive using the Cauchy-Schwarz inequality that $\left\|v_{H h}\right\|^{2} \leq\left\|u-\tilde{u}_{H h}\right\|\left\|v_{H h}\right\|$. Thus

$$
\left\|u_{H h}-\tilde{u}_{H h}\right\| \leq\left\|u-\tilde{u}_{H h}\right\| \text {. }
$$

With $u-u_{H h}=\left(u-\tilde{u}_{H h}\right)+\left(\tilde{u}_{H h}-u_{H h}\right)$ and (7), we have

$$
\left\|u-u_{H h}\right\| \leq\left\|u-\tilde{u}_{H h}\right\|+\left\|u_{H h}-\tilde{u}_{H h}\right\| \leq 2\left\|u-\tilde{u}_{H h}\right\| .
$$

Writing $u-\tilde{u}_{H h}=\left(\tilde{u}-\tilde{u}_{H}\right)+\left[(u-\tilde{u})-\tilde{u}_{h}\right]$, we get by standard interpolation results

$$
\begin{aligned}
\left\|u-\tilde{u}_{H h}\right\| & \leq\left\|\tilde{u}-\tilde{u}_{H}\right\|+\left\|(u-\tilde{u})-\tilde{u}_{h}\right\| \\
& \leq C\left(H^{r}\|\tilde{u}\|_{H^{q}(\Omega)}+h^{s}\|u-\tilde{u}\|_{H^{q}(\Lambda)}\right),
\end{aligned}
$$

and furthermore, with $\|u-\tilde{u}\|_{H^{q}(\Lambda)} \leq\|u\|_{H^{q}(\Lambda)}+\|\tilde{u}\|_{H^{q}(\Lambda)}$ and using the relations (5) and (6), we obtain

$$
\left\|u-\tilde{u}_{H h}\right\| \leq C\left(H^{r}\|u\|_{H^{q}(\Omega \backslash \bar{\Lambda})}+h^{s}\|u\|_{H^{q}(\Lambda)}\right) .
$$

Hence, combining the results (8) and (9) completes the proof.

As we have mentioned above, a priori $V_{H} \cap V_{h}$ does not necessarily reduce to the element zero and it is impossible, practically speaking, to exhibit a finite element basis of the space $V_{H h}$ and consequently to compute directly $u_{H h}$. It is the reason for which we suggest the following algorithm for computing $u_{H h}$. 
Algorithm 1 1. Set $u^{0}=u_{H} \in V_{H}$ and choose $\omega \in(0 ; 2)$.

2. For $n=1,2,3, \ldots$ find

(i) $w_{h} \in V_{h}$ such that $a\left(w_{h}, v\right)=\langle f \mid v\rangle-a\left(u^{n-1}, v\right), \quad \forall v \in V_{h}$; $u^{n-\frac{1}{2}}=u^{n-1}+\omega w_{h}$

(ii) $w_{H} \in V_{H}$ such that $a\left(w_{H}, v\right)=\langle f \mid v\rangle-a\left(u^{n-\frac{1}{2}}, v\right), \quad \forall v \in V_{H}$; $u^{n}=u^{n-\frac{1}{2}}+\omega w_{H}$.

When implementing the algorithm, the coarse and the fine parts of $u^{n}$ and $u^{n-\frac{1}{2}}$ are stored separately. In practice this is efficient for calculating the scalar product $a(\cdot, \cdot)$ in the right hand side of (i) and (ii).

It is readily seen that this algorithm is a Schwarz type domain decomposition method [33] with complete overlapping but without any conformity between the meshes $\mathcal{T}_{H}$ and $\mathcal{T}_{h}$ (see the work by Chan et al. [19]). This multiplicative Schwarz method is similar to the Gauss-Seidel method and is called by Xu successive subspace correction algorithm (see, e.g., [41]). The spaces $V_{H}$ and $V_{h}$ defined on the arbitrary triangulations $\mathcal{T}_{H}$ and $\mathcal{T}_{h}$ are not necessary orthognal nor share the only element zero as intersection. Note in particular that the sum which defines $V_{H h}$ is, a priori, not a direct sum. This property makes the above algorithm different from most known iterative schemes (see for example the scheme by Laydi [26]). The algorithm resembles the FAC method, see for example the works from McCormick et al. [29-31], or possibly a hierarchical method (see for example the papers from Yserentant [44,45], Bank et al. [10] and Bank and Smith [11]) with a mortar method (see [2]). It is also similar to the Chimera or overset grid method [17,35]. The new aspect we introduce here is to link the speed of convergence of this algorithm to the parameter $\tilde{\gamma}$, introduced here below, corresponding to an abstract angle between the spaces $V_{h}$ and $V_{H}$.

We shall now analyze the convergence of the two-scale algorithm.

If $P_{h}: V_{H h} \rightarrow V_{h}$ and $P_{H}: V_{H h} \rightarrow V_{H}$ are orthogonal projectors from $V_{H h}$ onto $V_{h}$ and $V_{H}$ respectively with regard to the scalar product $a(\cdot, \cdot)$, we have

$$
u_{H h}-u^{n}=\left(I-\omega P_{H}\right)\left(I-\omega P_{h}\right)\left(u_{H h}-u^{n-1}\right),
$$

where $I$ denotes the identity operator in $V_{H h}$. Setting

$$
B=\left(I-\omega P_{H}\right)\left(I-\omega P_{h}\right),
$$

we obtain that $u_{H h}-u^{n}=B^{n}\left(u_{H h}-u^{0}\right)$.

We set $V_{H h 0}=V_{H} \cap V_{h}$ and $V_{H h 0}^{\perp}$ the orthogonal complement of $V_{H h 0}$ in $V_{H h}$.

Proposition 2 If $\omega \in(0 ; 2)$, then the algorithm (i), (ii) converges, i.e. $\lim _{n \rightarrow \infty} \| u^{n}$ $-u_{H h} \|=0$. The convergence factor in the norm induced by the scalar product $a(\cdot, \cdot)$ is bounded by

$$
\|B\|=\frac{1}{2} \omega(2-\omega) \tilde{\gamma}+\sqrt{\frac{1}{4} \omega^{2}(2-\omega)^{2} \tilde{\gamma}^{2}+(\omega-1)^{2}}<1,
$$

where $\tilde{\gamma} \in[0 ; 1]$ is defined by

$$
\tilde{\gamma}= \begin{cases}\sup _{\substack{v_{h} \in V_{h} \cap V_{H h 0}^{\perp}, v_{h} \neq 0 \\ v_{H} \in V_{H} \cap V_{H h 0}^{\perp}, v_{H} \neq 0}} \frac{\left(v_{h}, v_{H}\right)}{\left\|v_{h}\right\|\left\|v_{H}\right\|}, & \text { if } V_{h} \neq V_{H h 0} \text { and } V_{H} \neq V_{H h 0}, \\ 0, & \text { otherwise. }\end{cases}
$$


We prove Proposition 2 at the end of Section 3 after studying some properties of vector spaces and doing an abstract analysis of the iteration operator $B$.

\section{Abstract analysis of the iteration operator $B$}

3.1 Theoretical preliminaries: some properties of vector spaces

Let $V$ be a Hilbert space with scalar product $(\cdot, \cdot)$ and denote by $\|\cdot\|$ the induced norm. Consider $V_{1}, V_{2}$ two closed subspaces of $V$.

We introduce the number

$$
\gamma= \begin{cases}\sup _{v_{1} \in V_{1}, v_{1} \neq 0} \frac{\left(v_{1}, v_{2}\right)}{\left\|v_{1} \mid\right\| v_{2} \|}, & \text { if } V_{1} \neq\{0\} \text { and } V_{2} \neq\{0\}, \\ 0, & \text { otherwise, }\end{cases}
$$

which is the constant from the corresponding strengthened C.B.S. inequality. The constant $\gamma$ is the cosine of the abstract angle between the two subspaces $V_{1}$ and $V_{2}$. We have the obvious properties for $\gamma$ :

1. Constant $\gamma$ is necessarily included in the interval $[0 ; 1]$.

2. If $V_{1} \cap V_{2} \neq\{0\}$, then we have $\gamma=1$.

3. Constant $\gamma=0$ if and only if $V_{1}$ is orthogonal to $V_{2}$.

We set $V_{0}=V_{1} \cap V_{2}$ and $V_{0}^{\perp}$ the orthogonal complement of $V_{0}$ in $V$. The second property suggests to introduce the number

$$
\tilde{\gamma}= \begin{cases}\sup _{\substack{v_{1} \in V_{1} \cap V_{0}^{\perp}, v_{1} \neq 0 \\ v_{2} \in V_{2} \cap V_{0}^{\perp}, v_{2} \neq 0}} \frac{\left(v_{1}, v_{2}\right)}{\left\|v_{1}\right\|\left\|v_{2}\right\|}, & \text { if } V_{1} \neq V_{0} \text { and } V_{2} \neq V_{0}, \\ 0, & \text { otherwise. }\end{cases}
$$

In the sequel we assume that the following hypothesis is satisfied:

Hypothesis $(\mathbf{H})$ There exists a constant $C_{0}$ such that for all $v \in V$ there exist $v_{1} \in V_{1}, v_{2} \in V_{2}$ satisfying $v=v_{1}+v_{2}$ and

$$
\left\|v_{1}\right\|^{2}+\left\|v_{2}\right\|^{2} \leq C_{0}^{2}\|v\|^{2} \text {. }
$$

Let us observe that:

1. If $(\mathrm{H})$ is satisfied, we have necessarily $V=V_{1}+V_{2}$.

2. If $V_{1} \neq V_{2}$, we have necessarily $C_{0} \geq 1$.

3. In the case $V_{1}=V_{2}=V$ the optimal constant $C_{0}$ in (13) is equal to $1 / \sqrt{2}$ (it suffices to take $v_{1}=v_{2}=\frac{1}{2} v, v \in V$ ).

4. If $V_{1}$ is orthogonal to $V_{2}$, we can take $C_{0}=1$ from Pythagore's Theorem.

Proposition 3 If $V=V_{1}+V_{2}$ then Hypothesis $(H)$ is satisfied and $\tilde{\gamma}<1$. If, moreover, $V_{1} \neq V_{2}$ then

$$
C_{0}^{\mathrm{opt}}=\sqrt{\frac{1}{1-\tilde{\gamma}}}
$$

is the optimal constant in (13). 
Proof Let us denote $\tilde{V}_{j}=V_{j} \cap V_{0}^{\perp}, j=1,2$, then $V_{0}^{\perp}=\tilde{V}_{1} \oplus \tilde{V}_{2}$ and $V=V_{0} \oplus$ $\tilde{V}_{1} \oplus \tilde{V}_{2}$. The Corollary of the Open Mapping Theorem (see Yosida [43], §II.5) for the one-to-one mapping $\left(\tilde{v}_{1}, \tilde{v}_{2}\right) \in \tilde{V}_{1} \times \tilde{V}_{2} \rightarrow \tilde{v}_{1}+\tilde{v}_{2} \in V_{0}^{\perp}$ yields the existence of $\tilde{C}_{0}<+\infty$ such that $\forall \tilde{v}_{j} \in \tilde{V}_{j}, j=1,2$, we have $\left\|\tilde{v}_{1}\right\|^{2}+\left\|\tilde{v}_{2}\right\|^{2} \leq \tilde{C}_{0}^{2}\left\|\tilde{v}_{1}+\tilde{v}_{2}\right\|^{2}$. We can take $\tilde{C}_{0} \geq 1$.

For all $v \in V$ we have a unique decomposition

$$
v=v_{0}+\tilde{v}_{1}+\tilde{v}_{2} \text { with } v_{0} \in V_{0}, \tilde{v}_{j} \in \tilde{V}_{j}, j=1,2 .
$$

Hence, we can put

$$
v_{1}=v_{0}+\tilde{v}_{1} \in V_{1} \text { and } v_{2}=\tilde{v}_{2} \in V_{2},
$$

so that $v=v_{1}+v_{2}$ and

$$
\begin{aligned}
\left\|v_{1}\right\|^{2}+\left\|v_{2}\right\|^{2} & =\left\|v_{0}\right\|^{2}+\left\|\tilde{v}_{1}\right\|^{2}+\left\|\tilde{v}_{2}\right\|^{2} \\
& \leq \tilde{C}_{0}^{2}\left(\left\|v_{0}\right\|^{2}+\left\|\tilde{v}_{1}+\tilde{v}_{2}\right\|^{2}\right)=\tilde{C}_{0}^{2}\|v\|^{2}
\end{aligned}
$$

i.e. Hypothesis $(\mathrm{H})$ is satisfied with $C_{0}=\tilde{C}_{0}$.

Let us now consider the case $V_{1} \neq V_{0}$ and $V_{2} \neq V_{0}$. Using Definition (12), there exists a sequence $v^{m}=\tilde{v}_{1}^{m}+\tilde{v}_{2}^{m}$ with $\tilde{v}_{1}^{m} \in \tilde{V}_{1}, \tilde{v}_{2}^{m} \in \tilde{V}_{2}$ and $\left\|\tilde{v}_{1}^{m}\right\|=\left\|\tilde{v}_{2}^{m}\right\|=1$ such that

$$
\left(\tilde{v}_{1}^{m}, \tilde{v}_{2}^{m}\right) \rightarrow-\tilde{\gamma}
$$

Suppose ad absurdum that $\tilde{\gamma}=1$. Thus

$$
\frac{\left\|\tilde{v}_{1}^{m}\right\|^{2}+\left\|\tilde{v}_{2}^{m}\right\|^{2}}{\left\|v^{m}\right\|^{2}}=\frac{1}{1+\left(\tilde{v}_{1}^{m}, \tilde{v}_{2}^{m}\right)} \rightarrow+\infty,
$$

which contradicts Hypothesis $(\mathrm{H})$. Hence $\tilde{\gamma}<1$.

Using again the decomposition (15) for any $v \in V$ and setting $v_{1} \in V_{1}$, $v_{2} \in V_{2}$ as in (16), we have $\left\|v_{1}\right\|^{2}+\left\|v_{2}\right\|^{2} \leq\left\|v_{0}\right\|^{2}+\left\|\tilde{v}_{1}+\tilde{v}_{2}\right\|^{2}+2\left|\left(\tilde{v}_{1}, \tilde{v}_{2}\right)\right| \leq$ $\|v\|^{2}+2 \tilde{\gamma}\left\|\tilde{v}_{1}\right\|\left\|\tilde{v}_{2}\right\|$. Since $2\left\|\tilde{v}_{1}\right\|\left\|\tilde{v}_{2}\right\| \leq\left\|\tilde{v}_{1}\right\|^{2}+\left\|\tilde{v}_{2}\right\|^{2} \leq\left\|v_{1}\right\|^{2}+\left\|v_{2}\right\|^{2}$, we get

$$
\left\|v_{1}\right\|^{2}+\left\|v_{2}\right\|^{2} \leq \frac{1}{1-\tilde{\gamma}}\|v\|^{2} .
$$

Thus we can choose $C_{0}=\sqrt{\frac{1}{1-\tilde{\gamma}}}$ in (13). It suffices to use (17) in (12) to show that $\sqrt{\frac{1}{1-\tilde{\gamma}}}$ is the best constant we can choose.

In the case $V_{1}=V_{0}$ or $V_{2}=V_{0}$ we have that $\tilde{\gamma}=0$ and if, moreover, $V_{1} \neq V_{2}$, then $C_{0}^{\text {opt }}=1$, i.e. (14) is also valid.

We introduce $P_{j}: V \rightarrow V_{j} \subset V$ the orthogonal projectors from $V$ upon $V_{j}$, $j=1,2$, and call $V_{j}^{\perp}$ the orthogonal complement of $V_{j}$ in $V$. 
Proposition 4 Let $V$ be of finite dimension and $V=V_{1}+V_{2}$. There exist $2 p$ $(p \geq 0)$ vectors $v_{1}^{(m)} \in V_{1}$ and $v_{2}^{(m)} \in V_{2}, m=1, \ldots, p$, such that

$$
\left\|v_{1}^{(m)}\right\|=\left\|v_{2}^{(m)}\right\|=1, \quad\left(v_{1}^{(m)}, v_{2}^{(m)}\right)=\gamma_{m}, \quad m=1, \ldots, p,
$$

with

$$
1>\gamma_{1} \geq \gamma_{2} \geq \cdots \geq \gamma_{p}>0
$$

and $V$ can be decomposed into the direct sum

$$
V=\left(V_{1} \cap V_{2}\right) \oplus\left(V_{1}^{\perp} \cap V_{2}\right) \oplus\left(V_{1} \cap V_{2}^{\perp}\right) \oplus L_{1} \oplus \cdots \oplus L_{p},
$$

where $L_{m}=\operatorname{span}\left\{v_{1}^{(m)}, v_{2}^{(m)}\right\}, m=1, \ldots, p$, and all the summands in (19) are mutually orthogonal subspaces of $V$, which are invariant with respect to both operators $P_{1}$ and $P_{2}$, i.e. $P_{j} L_{m} \subset L_{m}, j=1,2$.

Proof Let us prove that for any integer $k, 0 \leq k \leq p$ with $p$ to be identified later in the proof, the space $V$ can be decomposed into a direct sum with mutually orthogonal summands

$$
V=V_{0} \oplus W_{k} \oplus L_{1} \oplus \cdots \oplus L_{k}
$$

where $V_{0}=V_{1} \cap V_{2}$, the spaces $L_{m}$ are the two-dimensional subspaces of $V$ appearing in (19) and all the subspaces $V_{0}$ and $L_{1}, \ldots, L_{k}, W_{k} \subset V_{0}^{\perp}$ are invariant with respect to both operators $P_{1}$ and $P_{2}$. The decomposition (20) will be constructed by induction on $k$.

We start with $k=0$ and set $W_{0}=V_{0}^{\perp}$. Note that $V_{0}$ and $W_{0}$ are invariant subspaces of operators $P_{1}$ and $P_{2}$. On the $k$-th step of our construction $(k \geq 1)$ we suppose that (20) is established for $k-1$. Let $V_{1}^{(k)}=V_{1} \cap W_{k-1}, V_{2}^{(k)}=V_{2} \cap W_{k-1}$ and define

$$
\gamma_{k}= \begin{cases}\max _{\substack{v_{1} \in V_{1}^{(k)}, v_{2} \in V_{2}^{(k)} \\\left\|v_{1}\right\|=\left\|v_{2}\right\|=1}}\left(v_{1}, v_{2}\right), & \text { if } V_{1}^{(k)} \neq\{0\} \text { and } V_{2}^{(k)} \neq\{0\}, \\ 0, & \text { otherwise. }\end{cases}
$$

If $\gamma_{k}=0$ we stop the induction and set $p=k-1$. Indeed, it is easy to see that in this case, any vector from $V_{1}^{(k)}$ is orthogonal to $V_{2}$ and any vector from $V_{2}^{(k)}$ is orthogonal to $V_{1}$, i.e.

$$
W_{k-1} \subseteq\left(V_{1}^{\perp} \cap V_{2}\right) \oplus\left(V_{1} \cap V_{2}^{\perp}\right),
$$

which gives in combination with (20) the desired decomposition (19).

Assume now $\gamma_{k} \neq 0$ and let us construct $L_{k}$ and $W_{k}$. Note that $0<\gamma_{k}<1$. Indeed, if $\gamma_{k}=1$ there would exist a non-zero vector $v \in V_{1}^{(k)} \cap V_{2}^{(k)}=V_{1} \cap$ $V_{2} \cap W_{k-1} \subseteq V_{0} \cap V_{0}^{\perp}$, which is impossible. Let $v_{1}^{(k)} \in V_{1}^{(k)}$ and $v_{2}^{(k)} \in V_{2}^{(k)}$, $\left\|v_{1}^{(k)}\right\|=\left\|v_{2}^{(k)}\right\|=1$, be the vectors that give the maximum in (21) and $L_{k}=$ $\operatorname{span}\left\{v_{1}^{(k)}, v_{2}^{(k)}\right\}$. The vector $P_{1} v_{2}^{(k)}$ belongs to $V_{1}^{(k)}$ since $v_{2}^{(k)} \in W_{k-1}$ and $W_{k-1}$ is 
the invariant subspace of $P_{1}$ by induction hypothesis. Suppose that $P_{1} v_{2}^{(k)}$ is not parallel to $v_{1}^{(k)}$. We have then the inequality

$$
\left(v_{1}^{(k)}, v_{2}^{(k)}\right)=\left(v_{1}^{(k)}, P_{1} v_{2}^{(k)}\right)<\left\|P_{1} v_{2}^{(k)}\right\|=\left(\frac{P_{1} v_{2}^{(k)}}{\left\|P_{1} v_{2}^{(k)}\right\|}, v_{2}^{(k)}\right)
$$

which contradicts the definition of $v_{1}^{(k)}$ and $v_{2}^{(k)}$. This means that $P_{1} v_{2}^{(k)}$ is parallel to $v_{1}^{(k)}$, hence $P_{1} L_{k} \subset L_{k}$. One can prove in the same manner that $P_{2} v_{1}^{(k)}$ is parallel to $v_{2}^{(k)}$, hence $P_{2} L_{k} \subset L_{k}$. Let $W_{k}=\left(V_{0} \oplus W_{k-1} \oplus L_{1} \oplus \cdots \oplus L_{k}\right)^{\perp}$. The subspace $V_{0} \oplus W_{k-1} \oplus L_{1} \oplus \cdots \oplus L_{k}$ is invariant with respect to $P_{1}$ and $P_{2}$ and so is the subspace $W_{k}$ since operators $P_{1}$ and $P_{2}$ are symmetric.

Note at last that $W_{k-1}=W_{k} \oplus L_{k}$ hence for $k>1, V_{1}^{(k)} \subset V_{1}^{(k-1)}$ and $V_{2}^{(k)} \subset V_{2}^{(k-1)}$, i.e. $\gamma_{k} \leq \gamma_{k-1}$ according to (21). Thus we have result (18).

3.2 Norm and spectral radius of an operator involving the orthogonal projectors $P_{1}$ and $P_{2}$

If $\mathcal{L}(V)$ is the space of linear and continuous operators from $V$ into $V$, we denote by $\|B\|=\sup _{v \in V,\|v\|=1}\|B v\|$ the norm of $B \in \mathcal{L}(V)$. If $I$ denotes the identity operator in $V$ and $\omega$ is a real parameter, we define the operator $B \in \mathcal{L}(V)$ by

$$
B=\left(I-\omega P_{2}\right)\left(I-\omega P_{1}\right) .
$$

In this paragraph we formulate first a result for the norm of the operator $B$ in order to get an estimate as presented in [22]. The idea of Proposition 5 and its proof come originally from Bramble et al. [16]. In their work, an abstract analysis of product iterative methods is presented and similar convergence estimates are given. Comparable results proved using the technique from [16] can be found, for example, in early papers from $\mathrm{Xu}[39,40]$ and Yserentant [46] appended by the work of Griebel and Oswald [23], in the article of Cai and Widlund [18] or Wang [36], and in an abstract theory presented by Widlund in [37]. More recent reports include the framework of the successive subspace correction algorithm by Xu and Zikatanov [42] and $\mathrm{Xu}$ [41]. Some estimates in the framework of an abstract convergence analysis of Schwarz methods are presented in textbooks, e.g., by Quarteroni and Valli [32] (\$4.6), Smith et al. [34] (\$5.2) and Wohlmuth [38] (\$2.1).

Proposition 5 If Hypothesis $(H)$ is satisfied and if $0<\omega<2$, then the norm of the operator $B$ given by (22) verifies

$$
\|B\| \leq\left(1-\frac{(2-\omega) \omega}{C_{0}^{2}(1+\omega \gamma)^{2}}\right)^{\frac{1}{2}}<1 .
$$

Proof The proof is adapted from [16] to the present setting and we establish it for the convenience of the reader. Introduce $R_{1}=I-\omega P_{1}$ and $R_{2}=\left(I-\omega P_{2}\right)(I-$ $\left.\omega P_{1}\right)=B$. We begin by proving

$$
(2-\omega) \omega\left(\left\|P_{1} v\right\|^{2}+\left\|P_{2} R_{1} v\right\|^{2}\right)=\|v\|^{2}-\|B v\|^{2}, \quad \forall v \in V .
$$


As $v=R_{1} v+\omega P_{1} v,\|v\|^{2}=\left\|R_{1} v\right\|^{2}+\omega^{2}\left\|P_{1} v\right\|^{2}+2 \omega\left(R_{1} v, P_{1} v\right)$, and by definition $\left(R_{1} v, P_{1} v\right)=\left(\left(I-\omega P_{1}\right) v, P_{1} v\right)=(1-\omega)\left\|P_{1} v\right\|^{2}$. Hence

$$
\|v\|^{2}-\left\|R_{1} v\right\|^{2}=\left[\omega^{2}+2 \omega(1-\omega)\right]\left\|P_{1} v\right\|^{2}=(2-\omega) \omega\left\|P_{1} v\right\|^{2} .
$$

Furthermore, $R_{1} v=R_{2} v+\omega P_{2} R_{1} v$ implies $\left\|R_{1} v\right\|^{2}=\left\|R_{2} v\right\|^{2}+\omega^{2}\left\|P_{2} R_{1} v\right\|^{2}+$ $2 \omega\left(R_{2} v, P_{2} R_{1} v\right)$ and by definition $\left(R_{2} v, P_{2} R_{1} v\right)=\left(\left(I-\omega P_{2}\right) R_{1} v, P_{2} R_{1} v\right)=$ $(1-\omega)\left\|P_{2} R_{1} v\right\|^{2}$. Hence

$$
\left\|R_{1} v\right\|^{2}-\left\|R_{2} v\right\|^{2}=(2-\omega) \omega\left\|P_{2} R_{1} v\right\|^{2} .
$$

Summing (25) and (26), we get (24).

We next prove

$$
\left\|P_{1} v\right\|^{2}+\left\|P_{2} v\right\|^{2} \leq(1+\gamma \omega)^{2}\left(\left\|P_{1} v\right\|^{2}+\left\|P_{2} R_{1} v\right\|^{2}\right), \quad \forall v \in V .
$$

Starting from $I-R_{1}=\omega P_{1}$, we get

$$
\left(P_{2} v, v\right)-\left(P_{2} v, R_{1} v\right)=\omega\left(P_{2} v, P_{1} v\right),
$$

which implies that $\left\|P_{2} v\right\|^{2}=\left(P_{2} v, R_{1} v\right)+\omega\left(P_{2} v, P_{1} v\right)$. Hence

$$
\begin{aligned}
\left\|P_{1} v\right\|^{2}+\left\|P_{2} v\right\|^{2}= & \left(P_{1} v, P_{1} v\right)+\left(P_{2} v, P_{2} R_{1} v\right)+\omega\left(P_{2} v, P_{1} v\right) \\
\leq & \left(\left\|P_{1} v\right\|^{2}+\left\|P_{2} v\right\|^{2}\right)^{\frac{1}{2}}\left(\left\|P_{1} v\right\|^{2}+\left\|P_{2} R_{1} v\right\|^{2}\right)^{\frac{1}{2}} \\
& +\omega\left(P_{1} v, P_{2} v\right) .
\end{aligned}
$$

From the Definition (11) of $\gamma$ we get

$$
\begin{aligned}
\left|\left(P_{1} v, P_{2} v\right)\right| & \leq \gamma\left\|P_{1} v\right\|\left\|P_{2} v\right\| \leq \gamma\left(\left\|P_{2} v\right\|\left\|P_{1} v\right\|+\left\|P_{1} v\right\|\left\|P_{2} R_{1} v\right\|\right) \\
& \leq \gamma\left(\left\|P_{1} v\right\|^{2}+\left\|P_{2} v\right\|^{2}\right)^{\frac{1}{2}}\left(\left\|P_{1} v\right\|^{2}+\left\|P_{2} R_{1} v\right\|^{2}\right)^{\frac{1}{2}} .
\end{aligned}
$$

Thus we have

$$
\begin{aligned}
& \left\|P_{1} v\right\|^{2}+\left\|P_{2} v\right\|^{2} \\
& \quad \leq(1+\omega \gamma)\left(\left\|P_{1} v\right\|^{2}+\left\|P_{2} v\right\|^{2}\right)^{\frac{1}{2}}\left(\left\|P_{1} v\right\|^{2}+\left\|P_{2} R_{1} v\right\|^{2}\right)^{\frac{1}{2}},
\end{aligned}
$$

which leads to (27).

Finally, we show that Hypothesis $(\mathrm{H})$ implies

$$
\|v\|^{2} \leq C_{0}^{2}\left(\left\|P_{1} v\right\|^{2}+\left\|P_{2} v\right\|^{2}\right), \quad \forall v \in V .
$$

When $v \in V$, there exist $v_{1} \in V_{1}, v_{2} \in V_{2}$ such that $v=v_{1}+v_{2}$ and $\left\|v_{1}\right\|^{2}+$ $\left\|v_{2}\right\|^{2} \leq C_{0}^{2}\|v\|^{2}($ see Hypothesis $(\mathrm{H}))$. Hence $\|v\|^{2}=\left(v_{1}, v\right)+\left(v_{2}, v\right)=$ $\left(v_{1}, P_{1} v\right)+\left(v_{2}, P_{2} v\right)$. Result (28) thus follows from:

$$
\begin{aligned}
\|v\|^{2} & \leq\left\|v_{1}\right\|\left\|P_{1} v\right\|+\left\|v_{2}\right\|\left\|P_{2} v\right\| \\
& \leq\left(\left\|v_{1}\right\|^{2}+\left\|v_{2}\right\|^{2}\right)^{\frac{1}{2}}\left(\left\|P_{1} v\right\|^{2}+\left\|P_{2} v\right\|^{2}\right)^{\frac{1}{2}} \\
& \leq C_{0}\|v\|\left(\left\|P_{1} v\right\|^{2}+\left\|P_{2} v\right\|^{2}\right)^{\frac{1}{2}} .
\end{aligned}
$$


The proof of Proposition 5 is now straightforward. Combining (24) and (27), we get for all $v \in V$,

$$
\frac{(2-\omega) \omega}{(1+\gamma \omega)^{2}}\left(\left\|P_{1} v\right\|^{2}+\left\|P_{2} v\right\|^{2}\right) \leq\|v\|^{2}-\|B v\|^{2}
$$

and finally, (28) yields

$$
\frac{(2-\omega) \omega}{C_{0}^{2}(1+\gamma \omega)^{2}}\|v\|^{2} \leq\|v\|^{2}-\|B v\|^{2}
$$

Thus $\|B v\|^{2} \leq\left(1-\frac{(2-\omega) \omega}{C_{0}^{2}(1+\gamma \omega)^{2}}\right)\|v\|^{2}$, i.e. $\|B\| \leq\left(1-\frac{(2-\omega) \omega}{C_{0}^{2}(1+\omega \gamma)^{2}}\right)^{\frac{1}{2}}$ which is strictly bounded by one if $0<\omega<2$.

It is readily seen that the estimate of Proposition 5 is not optimal even in the case where $V=V_{1} \oplus V_{2}$. In particular, if the space $V$ is two-dimensional and $V_{1}$ and $V_{2}$ are one-dimensional subspaces of $V$, then $\|B\|=\gamma$ for $\omega=1$. Indeed, $\forall v \in V$ we have in this case $\|B v\|^{2}=\left|\left(B v,\left(I-P_{1}\right) v\right)\right|=\gamma\|B v\|\left\|\mid\left(I-P_{1}\right) v\right\|$ since $\left(I-P_{1}\right) v \in V_{1}^{\perp}, B v \in V_{2}^{\perp}$ and the angle between $V_{1}^{\perp}$ and $V_{2}^{\perp}$ is equal to the angle between $V_{1}$ and $V_{2}$. However, estimate (23) with the best choice of $C_{0}$ (14) gives only $\|B\| \leq \sqrt{\gamma(\gamma+3)} /(1+\gamma)$, which is optimal only if $\gamma=0$. The non-optimality of (23) is also discussed, for example, by Griebel and Oswald in the concluding remarks of [23].

In the case where $V_{1}$ and $V_{2}$ are of finite dimension, an analysis of the spectral properties of $B$ leads to exact formulas for its spectral radius and its norm. Hereafter we present these new results.

For $\tilde{\gamma}$ and $\omega \in(0 ; 2)$ we define the functions

$$
\rho(\tilde{\gamma}, \omega)= \begin{cases}\frac{\omega^{2} \tilde{\gamma}^{2}}{2}-\omega+1+\frac{\omega \tilde{\gamma}}{2} \sqrt{\omega^{2} \tilde{\gamma}^{2}-4 \omega+4,}, & \text { if } \omega \leq \omega_{0}(\tilde{\gamma}), \\ \omega-1, & \text { otherwise }\end{cases}
$$

where

$$
\omega_{0}(\tilde{\gamma})= \begin{cases}\frac{2-2 \sqrt{1-\tilde{\gamma}^{2}}}{\tilde{\gamma}^{2}}, & \text { for } \tilde{\gamma} \in(0 ; 1] \\ 1, & \text { for } \tilde{\gamma}=0\end{cases}
$$

and

$$
N(\tilde{\gamma}, \omega)=\frac{1}{2} \omega(2-\omega) \tilde{\gamma}+\sqrt{\frac{1}{4} \omega^{2}(2-\omega)^{2} \tilde{\gamma}^{2}+(\omega-1)^{2}}
$$

Proposition 6 Let $V$ be offinite dimension, $V=V_{1}+V_{2}$ and $\tilde{\gamma}$ be defined by (12). The spectral radius of operator $B$ given by (22) is a function of $\tilde{\gamma}$ and $\omega \in(0 ; 2)$ given by $\rho(B)=\rho(\tilde{\gamma}, \omega)$. The norm of $B$ is a function of $\tilde{\gamma}$ and $\omega \in(0 ; 2)$ given by $\|B\|=N(\tilde{\gamma}, \omega)$. 
Proof The idea of the proof is to establish first all the results in the two-dimensional case and to use then decomposition (19) to extend the results to the general case. Therefore, we assume first that the space $V$ is two-dimensional and $V_{1}$ and $V_{2}$ are one-dimensional subspaces of $V$ spanned by the vectors $v_{1}$ and $v_{2}$, respectively. Without loss of generality, we can assume that $\left\|v_{1}\right\|=\left\|v_{2}\right\|=1$ and $\left(v_{1}, v_{2}\right)=\tilde{\gamma}$. We can verify that the linear operator $B$ is represented in the basis $\left\{v_{1}, v_{2}\right\}$ by the matrix

$$
\mathbf{B}=\left(\begin{array}{cc}
1-\omega & -\omega \tilde{\gamma} \\
\omega(\omega-1) \tilde{\gamma} & \omega^{2} \tilde{\gamma}^{2}+1-\omega
\end{array}\right) .
$$

The characteristic polynomial of this matrix is

$$
p(\lambda)=\lambda^{2}-\left(\omega^{2} \tilde{\gamma}^{2}-2 \omega+2\right) \lambda+(\omega-1)^{2} .
$$

If $\tilde{\gamma}>0$ and $\omega \in\left(\omega_{0}(\tilde{\gamma}) ; 2\right), p(\lambda)$ has two complex conjugate roots $\lambda_{ \pm}$such that $\left|\lambda_{ \pm}\right|=\omega-1$. If $\tilde{\gamma}>0$ and $\omega \in\left(0 ; \omega_{0}(\tilde{\gamma})\right), p(\lambda)$ has two real roots $\lambda_{ \pm}$given by

$$
\lambda_{ \pm}=\frac{\omega^{2} \tilde{\gamma}^{2}}{2}-\omega+1 \pm \frac{\omega \tilde{\gamma}}{2} \sqrt{\omega^{2} \tilde{\gamma}^{2}-4 \omega+4}
$$

If $\tilde{\gamma}=0, p(\lambda)$ has the only double root $\lambda=1-\omega$. Identity $\rho(B)=\rho(\tilde{\gamma}, \omega)$ is thus proved in the two-dimensional case.

Let us consider now the norm of operator $B$ that can be written as

$$
\|B\|^{2}=\max _{x \in \mathbb{R}^{2}, x \neq 0} \frac{x^{T} \mathbf{B}^{T} \Gamma \mathbf{B} x}{x^{T} \Gamma x},
$$

where $\Gamma$ is the Gramm matrix of the basis $\left\{v_{1}, v_{2}\right\}$,

$$
\Gamma=\left(\begin{array}{cc}
1 & \tilde{\gamma} \\
\tilde{\gamma} & 1
\end{array}\right) \text {. }
$$

By making the substitution $y=\Gamma^{1 / 2} x$, we can rewrite (31) as

$$
\|B\|^{2}=\max _{y \in \mathbb{R}^{2}, y \neq 0} \frac{y^{T} \Gamma^{-1 / 2} \mathbf{B}^{T} \Gamma \mathbf{B} \Gamma^{-1 / 2} y}{y^{T} y} .
$$

Since the matrix $\mathbf{C}=\Gamma^{-1 / 2} \mathbf{B}^{T} \Gamma \mathbf{B} \Gamma^{-1 / 2}$ is symmetric positive definite, (32) implies that $\|B\|^{2}$ is equal to the spectral radius of $\mathbf{C}$. Let $\mu^{2}$ be an eigenvalue of $\mathbf{C}$, then

$$
\operatorname{det}\left(\mathbf{C}-\mu^{2} \mathbf{I}\right)=0
$$

But

$$
\begin{aligned}
& \operatorname{det}\left(\mathbf{C}-\mu^{2} \mathbf{I}\right) \\
& \quad=\operatorname{det}\left(\mathbf{B}^{T} \Gamma \mathbf{B} \Gamma^{-1}-\mu^{2} \mathbf{I}\right) \\
& \quad=\mu^{4}-\mu^{2} \operatorname{tr}\left(\mathbf{B}^{T} \Gamma \mathbf{B} \Gamma^{-1}\right)+\operatorname{det}\left(\mathbf{B}^{T} \Gamma \mathbf{B} \Gamma^{-1}\right) \\
& \quad=\mu^{4}-\mu^{2}\left[(2-\omega)^{2} \omega^{2} \tilde{\gamma}^{2}+2(\omega-1)^{2}\right]+(\omega-1)^{4} \\
& \quad=\left(\mu^{2}-\omega(2-\omega) \tilde{\gamma} \mu-(\omega-1)^{2}\right)\left(\mu^{2}+\omega(2-\omega) \tilde{\gamma} \mu-(\omega-1)^{2}\right) .
\end{aligned}
$$


The roots of (33) are thus given by

$$
\mu= \pm \frac{1}{2} \omega(2-\omega) \tilde{\gamma} \pm \sqrt{\frac{1}{4} \omega^{2}(2-\omega)^{2} \tilde{\gamma}^{2}+(\omega-1)^{2}},
$$

and the largest among them gives $\|B\|$, i.e. identity $\|B\|=N(\tilde{\gamma}, \omega)$ is proved in the two-dimensional case.

Let us turn now to the general case. According to Proposition $4, V$ can be decomposed into the direct sum (19) where all the summands are invariant subspaces of projectors $P_{1}$ and $P_{2}$, and hence of $B$. Hence the spectrum of $B$ is given by the set of all eigenvalues of the operators $B_{0}=\left.B\right|_{V_{0}}, B_{12}=\left.B\right|_{V_{1}^{\perp} \cap V_{2}}, B_{21}=\left.B\right|_{V_{1} \cap V_{2}^{\perp}}$ and $B_{m}=\left.B\right|_{L_{m}}, m=1,2, \ldots, p$, where here $\left.B\right|_{W}$ is the restriction of $B$ to $W$. We verify easily that $\rho\left(B_{0}\right)=(1-\omega)^{2}, \rho\left(B_{12}\right)=\rho\left(B_{21}\right)=|1-\omega|$, and concerning the two-dimensional spaces $L_{m}, m=1,2, \ldots, p$, we have proved just above that $\rho\left(B_{m}\right)=\rho\left(\gamma_{m}, \omega\right)$ where $\rho(\gamma, \omega)$ is defined by (29). Hence

$$
\rho(B)=\max \left((1-\omega)^{2},|1-\omega|, \rho\left(\gamma_{1}, \omega\right), \ldots, \rho\left(\gamma_{p}, \omega\right)\right) .
$$

It is easy to verify that $\omega_{0}(\gamma)$ is an increasing function and for fixed $\omega, \rho(\gamma, \omega)$ is a non-decreasing function. It follows that we have $\rho\left(\gamma_{1}, \omega\right) \geq \cdots \geq \rho\left(\gamma_{p}, \omega\right)>$ $\rho(0, \omega)=|1-\omega|$. Since $\tilde{\gamma}=\gamma_{1}$ if $p>0$ and $\tilde{\gamma}=0$ if $p=0$, we conclude that $\rho(B)=\rho(\tilde{\gamma}, \omega)$. Analogously, since all the subspaces in (19) are mutually orthogonal, Pythagore's Theorem implies

$$
\|B\|=\max \left((1-\omega)^{2},|1-\omega|, N\left(\gamma_{1}, \omega\right), \ldots, N\left(\gamma_{p}, \omega\right)\right),
$$

where $N(\gamma, \omega)$ is defined by (30). Noting that $N(0, \omega)=|1-\omega|$, we conclude that $\|B\|=N(\tilde{\gamma}, \omega)$.

Finally, let us observe that:

1. The spectral radius $\rho(B)$ is less than one for $\omega \in(0 ; 2)$ and, for $\tilde{\gamma}$ given by (12), attains the minimum value $\rho(B)=\omega_{0}(\tilde{\gamma})-1$ at $\omega=\omega_{0}(\tilde{\gamma}) \in[1 ; 2)$. We have $\rho(B)=\tilde{\gamma}^{2}$ at $\omega=1$.

2. The norm $\|B\|$ is less than one for $\omega \in(0 ; 2)$ and, for $\tilde{\gamma}$ given by (12), attains the minimum value $\|B\|=\tilde{\gamma}$ at $\omega=1$. This last result is given by Blaheta in [12].

3. The functions $\rho(\tilde{\gamma}, \omega)$ and $N(\tilde{\gamma}, \omega)$ are non-decreasing with respect to $\tilde{\gamma}$ for any fixed value of $\omega \in(0 ; 2)$.

4. Both formulas (29) and (30) can be rewritten in the case $V_{1} \neq V_{2}$ as the functions only of $C_{0}^{\mathrm{opt}}$ and $\omega$ due to the relation (14).

\subsection{Proof of Proposition 2}

The above abstract analysis enables us to prove Proposition 2 . This is readily done by applying Proposition 6 to $V=V_{H h}, V_{1}=V_{h}$ and $V_{2}=V_{H}$ using the form $a(\cdot, \cdot)$ as scalar product. 


\section{Estimates for $\gamma$}

Estimates and upper bounds for the constant from the C.B.S. inequality are abundant in the literature as it is the main tool in the convergence analysis of many methods. The C.B.S. inequality has been used in two-level methods by Axelsson [4], Axelsson and Gustavson [7], Braess [13, 14], Maître and Musy [27]. A survey of the role of this constant is reported by Axelsson and Vassilevski [8,9] and by Eijkhout and Vassilevski [21]. The constant is also used in local refinement preconditioning methods, e.g., by McCormick [29] and Bramble et al. [15]. The latest papers present estimates of $\gamma$ depending generally on the bilinear form $a$, i.e. on the problem coefficients, and the type and shape of the finite element used. In some cases it is possible to have universal bounds [6]. Margenov [28] gives estimates of the $2 \mathrm{D}$ elasticity problem on a triangular mesh. More recently Achchab and Maître [1] and Axelsson [5] proved that the constant $\gamma^{2}$ is bounded from above by 3/4 for the 2D elasticity problem on a triangular mesh. Numerical experiments by Jung and Maître [25] generalize the latter to more choices of finite elements.

Let $a_{i j} \in W^{1, \infty}(\Omega), 1 \leq i, j \leq 2$, verifying $a_{i j}=a_{j i}$ and the hypothesis of strong ellipticity,

$$
\sum_{i, j=1}^{2} a_{i j}(x) \xi_{i} \xi_{j} \geq \alpha \sum_{i=1}^{2} \xi_{i}^{2}, \quad \forall\left(\xi_{1}, \xi_{2}\right) \in \mathbb{R}^{2}, \text { a.e. in } \Omega
$$

where $\alpha$ is a positive constant. If $\mathcal{L}$ is the elliptic operator given by

$$
\mathcal{L}(u)=-\sum_{i, j=1}^{2} \frac{\partial}{\partial x_{i}}\left(a_{i j} \frac{\partial u}{\partial x_{j}}\right),
$$

the associated bilinear form is given by

$$
a(u, v)=\sum_{i, j=1}^{2} \int_{\Omega} a_{i j} \frac{\partial u}{\partial x_{j}} \frac{\partial v}{\partial x_{i}} \mathrm{~d} \mathbf{x} .
$$

We consider the case with $\bar{\Lambda} \subset K$, for $K \in \mathcal{T}_{H}$. Let $\tilde{\Lambda} \supseteq \Lambda$ be a rectangle with dimensions $L_{1}$ and $L_{2}$ and define

$$
\tilde{\lambda}=\min _{v \in H_{0}^{1}(\tilde{\Lambda}), v \neq 0}\|\nabla v\|_{L^{2}(\tilde{\Lambda})}^{2} /\|v\|_{L^{2}(\tilde{\Lambda})}^{2} .
$$

We have $\tilde{\lambda}=\pi^{2}\left(1 / L_{1}^{2}+1 / L_{2}^{2}\right)$ and we introduce $d=\sqrt{1 / \tilde{\lambda}}$. We set

$$
\beta=\left[\sum_{j=1}^{2}\left(\sum_{i=1}^{2}\left\|\frac{\partial a_{i j}}{\partial x_{i}}\right\|_{L_{(\Lambda)}^{\infty}}\right)^{2}\right]^{\frac{1}{2}}
$$

Proposition 7 If (34) is satisfied and if there exists $K \in \mathcal{T}_{H}$ such that $\bar{\Lambda} \subset K$ and if $r=1$, then $\gamma \leq \frac{\beta d}{\alpha}$. If furthermore the $a_{i j}$ 's are constant over $\Lambda, 1 \leq i, j \leq 2$, the Algorithm 1 converges in only one iteration when $\omega=1$. 
Proof We shall first prove that $\gamma \leq \frac{\beta d}{\alpha}$. For any $u_{H} \in V_{H}, v_{h} \in V_{h}$, we have

$$
\left|a\left(u_{H}, v_{h}\right)\right|=\left|\sum_{i, j=1}^{2} \int_{\Lambda} a_{i j} \frac{\partial u_{H}}{\partial x_{j}} \frac{\partial v_{h}}{\partial x_{i}} \mathrm{~d} \mathbf{x}\right|,
$$

as $v_{h}=0$ in $\Omega \backslash \bar{\Lambda}$. Since $\bar{\Lambda} \subset K \in \mathcal{T}_{H}, \frac{\partial u_{H}}{\partial x_{j}}$ is constant over $\bar{\Lambda}$ so that

$$
\left|a\left(u_{H}, v_{h}\right)\right|=\left|\sum_{i, j=1}^{2} \frac{\partial u_{H}}{\partial x_{j}}\right|_{K} \int_{\Lambda} \frac{\partial a_{i j}}{\partial x_{i}} v_{h} \mathrm{~d} \mathbf{x} \mid,
$$

where we have applied the divergence theorem taking into account that $v_{h}=0$ on $\partial \Lambda$. By the Cauchy-Schwarz inequality we have

$$
\begin{aligned}
\left|a\left(u_{H}, v_{h}\right)\right| & \leq \sum_{i, j=1}^{2}\left\|\frac{\partial a_{i j}}{\partial x_{i}}\right\|_{L^{\infty}(\Lambda)}\left|\frac{\partial u_{H}}{\partial x_{j}}\right|_{K} \int_{\Lambda}\left|v_{h}\right| \mathrm{d} \mathbf{x} \mid \\
& \leq \beta\left(\sum_{j=1}^{2}\left\|\frac{\partial u_{H}}{\partial x_{j}}\right\|_{L^{2}(\Lambda)}^{2}\left\|v_{h}\right\|_{L^{2}(\Lambda)}^{2}\right)^{\frac{1}{2}} \\
& =\beta\left\|\nabla u_{H}\right\|_{L^{2}(\Lambda)}\left\|v_{h}\right\|_{L^{2}(\Lambda)} .
\end{aligned}
$$

At this point we need to bound $\left\|v_{h}\right\|_{L^{2}(\Lambda)}$ from above with $\left\|\nabla v_{h}\right\|_{L^{2}(\Lambda)}$. We introduce $\lambda=\min _{v \in H_{0}^{1}(\Lambda), v \neq 0}\|\nabla v\|_{L^{2}(\Lambda)}^{2} /\|v\|_{L^{2}(\Lambda)}^{2}$, the smallest value of the Rayleigh quotient. In order to estimate $\lambda$, we consider the rectangle $\tilde{\Lambda}$ and $\tilde{\lambda}$ as introduced above. As $\Lambda \subseteq \tilde{\Lambda}$ we have $\lambda \geq \tilde{\lambda}=1 / d^{2}$, i.e. we get $\left\|v_{h}\right\|_{L^{2}(\Lambda)} \leq$ $d\left\|\nabla v_{h}\right\|_{L^{2}(\Lambda)}$. Hence combining the previous results,

$$
\left|a\left(u_{H}, v_{h}\right)\right| \leq \beta d|| \nabla u_{H}\left\|_{L^{2}(\Lambda)}|| \nabla v_{h}\right\|_{L^{2}(\Lambda)} .
$$

The hypothesis of strong ellipticity (34) implies that, $\forall u \in H_{0}^{1}(\Omega)$,

$$
\begin{aligned}
a(u, u) & =\int_{\Omega} \sum_{i, j=1}^{2} a_{i j} \frac{\partial u}{\partial x_{j}} \frac{\partial u}{\partial x_{i}} \mathrm{~d} \mathbf{x} \\
& \geq \alpha\|\nabla u\|_{L^{2}(\Omega)}^{2},
\end{aligned}
$$

i.e. $\alpha\|\nabla u\|_{L^{2}(\Lambda)}^{2} \leq \alpha\|\nabla u\|_{L^{2}(\Omega)}^{2} \leq a(u, u)=\|u\|^{2}$. Applying this inequality to $u_{H}$ and $v_{h}$, we obtain $\left|a\left(u_{H}, v_{h}\right)\right| \leq \frac{\beta d}{\alpha}|| u_{H}|||| v_{h} \|$, i.e. $\gamma \leq \frac{\beta d}{\alpha}$.

If the $a_{i j}$ 's are constant over $\Lambda, 1 \leq i, j \leq 2$, we clearly have $\beta=0$, thus $\gamma=0$ and $C_{0}=1$. In this case $V_{H}$ and $V_{h}$ are orthogonal and, since $B=0$ for $\omega=1$, the algorithm converges in only one iteration. 

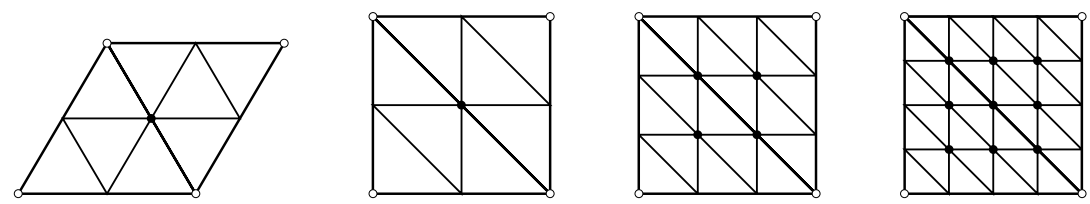

Fig. 2 Illustration of the triangulations of $\Lambda$ considered in Table 1. White dots refer to the degrees of freedom of $r_{H} v$, black dots refer to those of $v-r_{H} v$

Table 1 Upper bounds for $\gamma$

\begin{tabular}{lll}
\hline Triangles & $H / h$ & Upper bound for $\gamma$ \\
\hline equilateral & 2 & $\sqrt{3} / 3 \approx 0.577$ \\
right isosceles & 2 & $\sqrt{2} / 2 \approx 0.707$ \\
right isosceles & 3 & $2 / 3 \approx 0.667$ \\
right isosceles & 4 & $\sqrt{2} / 2 \approx 0.707$ \\
\hline
\end{tabular}

In the case where $\Lambda \subset K_{1} \cup K_{2}$, with $K_{1}, K_{2} \in \mathcal{T}_{H}$, the analysis gets more complicated. In the sequel we present some upper bounds for $\gamma$ in the case where $a_{i j}=\delta_{i j}$, i.e. $a(u, v)=\int_{\Omega} \nabla u \cdot \nabla v \mathrm{~d} \mathbf{x}$, and with $\Lambda$ the union of two triangles $K_{1}$ and $K_{2}$ of $\mathcal{T}_{H}, \mathcal{T}_{h}$ conforming with $\mathcal{T}_{H}$ and $r=s=1$. We consider the situations as illustrated in Figure 2 by the triangulations of the patch $\Lambda$. Estimates can be obtained by splitting $v \in V_{H h}$ into $v=v_{h}+v_{H}$, where $v_{H}=r_{H} v$ is the interpolant of $v$ in $V_{H}$ and $v_{h}=v-r_{H} v \in V_{h}$. The degrees of freedom of $v_{h}$ and $v_{H}$ in $\bar{\Lambda}$ are depicted in Figure 2. Using the fact that $v_{h}=0$ in $\Omega \backslash \bar{\Lambda}$ and the divergence theorem, we have that

$$
a\left(v_{H}, v_{h}\right) \leq\left|\left[\frac{\partial v_{H}}{\partial n}\right]_{\Gamma}\right| \int_{\Gamma}\left|v_{h}\right| \mathrm{d} s
$$

where $\Gamma=\partial K_{1} \cap \partial K_{2},[\cdot]_{\Gamma}$ denotes the jump on $\Gamma$ in the direction of a normal unit vector $\mathbf{n}$ on $\Gamma$. The first factor of the right-hand side of (35) can be bounded by

$$
\left|\left[\frac{\partial v_{H}}{\partial n}\right]_{\Gamma}\right| \leq \sum_{i=1}^{2} \frac{\left\|\nabla v_{H}\right\|_{L^{2}\left(K_{i}\right)}}{\sqrt{\operatorname{area}\left(K_{i}\right)}} \leq \frac{\sqrt{2}}{\min _{i=1,2} \sqrt{\operatorname{area}\left(K_{i}\right)}}\left\|\nabla v_{H}\right\|_{L^{2}(\Lambda)},
$$

and $\left\|\nabla v_{H}\right\|_{L^{2}(\Lambda)} \leq\left\|\nabla v_{H}\right\|_{L^{2}(\Omega)}$. As the dimension of $V_{h}$ is small in our cases, we evaluate $\int_{\Gamma}\left|v_{h}\right| \mathrm{d} s$ explicitely, and express it in relation to $\left\|\nabla v_{h}\right\|_{L^{2}(\Lambda)}=$ $\left\|\nabla v_{h}\right\|_{L^{2}(\Omega)}$. Applying the above procedure to our situations, we get $a\left(v_{H}, v_{h}\right) \leq$ $C\left\|v_{H}\right\|\left\|v_{h}\right\|$ and hence we have $\gamma \leq C$. The upper bounds found for $\gamma$ are reported in Table 1 . Note that the bound for $\gamma$ on right isosceles triangles with $H / h=2$ is reported by Axelsson and Gustafsson in [7].

The result of Proposition 6 with (29) gives an algebraic relationship of the spectral radius $\rho$ of the operator $B$ as function of $\tilde{\gamma}$ and $\omega$. This leads to a very convenient application to determine numerically a good approximation for $\tilde{\gamma}$. Running Algorithm 1 for given $\omega$ we can evaluate numerically an estimate of $\rho$ and hence find an estimate of the parameter $\tilde{\gamma}$. A study of $\tilde{\gamma}$ for various spaces $V_{1}=V_{h}$ and 
Table 2 Numerical estimates for $\gamma$

\begin{tabular}{lcc}
\hline Triangles & $H / h$ & Numerical estimate for $\gamma$ \\
\hline right isosceles & 2 & 0.426 \\
right isosceles & 3 & 0.464 \\
right isosceles & 4 & 0.476 \\
\hline
\end{tabular}

Table 3 Estimates for $\tilde{\gamma}$ for some patches and grids

\begin{tabular}{lllll}
\hline$H / h$ & 2 & 4 & 8 & 16 \\
\hline$\tilde{\gamma}$ & 0.273 & 0.306 & 0.313 & 0.315 \\
\hline
\end{tabular}

(a) Estimates for $\tilde{\gamma}$ for varying $H / h$ in the structured nested case $(k=0)$ with $N=8$.

\begin{tabular}{llll}
\hline$N$ & 8 & 16 & 32 \\
\hline$\tilde{\gamma}$ & 0.306 & 0.461 & 0.50 \\
\hline
\end{tabular}

(b) Estimates for $\tilde{\gamma}$ for varying $N$ in the structured nested case $(k=0)$ with $H / h=4$.

\begin{tabular}{llllllllll}
\hline$k$ & 0 & 0.5 & 1 & 1.5 & 2 & 2.5 & 3 & 3.5 & 4 \\
\hline$\tilde{\gamma}$ & 0.306 & 0.915 & 0.908 & 0.812 & 0.785 & 0.812 & 0.908 & 0.915 & 0.474 \\
\hline
\end{tabular}

(c) Estimates for $\tilde{\gamma}$ for different non-nested cases of structured grids with $N=8$ and $H / h=4$.

\begin{tabular}{lllll}
\hline$H / h$ & 2 & 4 & 8 & 16 \\
\hline$\tilde{\gamma}$ & 0.920 & 0.944 & 0.947 & 0.970 \\
\hline
\end{tabular}

(d) Estimates for $\tilde{\gamma}$ for varying $H / h$ in the unstructured case with $N=8$

$V_{2}=V_{H}$ is presented in $\S 6.1$. Estimates for the parameter $\gamma$ corresponding to the situations of Figure 2 with right isosceles triangles are given in Table 2.

\section{Generalization to multiple spaces}

The objective of this section is to generalize the two-scale algorithm presented in Section 2 to more than one level of refinement.

We consider again problem (1). We approximate $u$ by a finite element method. Introduce a triangulation $\mathcal{T}_{H}$ of $\bar{\Omega}$, define $V_{H}$ by (2) and calculate $u_{H} \in V_{H}$ satisfying equation (3).

Consider now $\Lambda_{j} \subset \Omega, j=1, \ldots, N-1$, other polygonal domains wherein we would like to obtain a better precision on the solution $u$ than the one given by $u_{H}$. Take note that $\bar{\Lambda}_{j}$ is not necessarily the union of several triangles $K$ of $\mathcal{T}_{H}$. Let $\mathcal{T}_{h_{j}}, j=1, \ldots, N-1$, be a triangulation of $\bar{\Lambda}_{j}$ and consider for $j=1, \ldots, N-1$,

$$
\begin{aligned}
& V_{h_{j}}=\left\{g: \bar{\Omega} \rightarrow \mathbb{R} \text { continuous such that }\left.g\right|_{K} \in \mathbb{P}_{s_{j}}(K), \forall K \in \mathcal{T}_{h_{j}}\right. \\
& \left.\quad \text { and } g=0 \text { on } \bar{\Omega} \backslash \Lambda_{j}\right\} .
\end{aligned}
$$


Call $\mathcal{T}_{h_{N}}=\mathcal{T}_{H}$ and $V_{h_{N}}=V_{H}$. Setting $V_{H h}=\sum_{j=1}^{N} V_{h_{j}}$ we search as approximation for $u$ the function $u_{H h} \in V_{H h}$ satisfying the equation (4).

The intersection $V_{i} \cap V_{j}$, for any $1 \leq i, j \leq N$, does not necessarily reduce to the element zero, making it impossible to explicit a finite element basis of the space $V_{H h}$. The generalization of Algorithm 1 to compute $u_{H h}$ is the following:

\section{Algorithm 2}

1. Set $u^{0}=u_{H} \in V_{H}$ and choose $\omega \in(0 ; 2)$.

2. For $n=1,2,3, \ldots$ find

$$
\mid \begin{aligned}
& \text { for } j=1,2, \ldots, N, \\
& \quad \begin{array}{l}
a\left(w_{h_{j}}, v\right)=\langle f \mid v\rangle-a\left(u^{n-1+\frac{j-1}{N}}, v\right), \quad \forall v \in V_{h_{j}} \\
w_{h_{j}} \in V_{h_{j}} \text { such that }
\end{array} \\
& u^{n-1+\frac{j}{N}}=u^{n-1+\frac{j-1}{N}}+\omega w_{h_{j}} .
\end{aligned}
$$

If $P_{h_{j}}: V_{H h} \rightarrow V_{h_{j}}, j=1,2, \ldots, N$, are orthogonal projectors from $V_{H h}$ to $V_{h_{j}}$ with respect to the scalar product $a(\cdot, \cdot)$, it is easy to verify that $u_{H h}-u^{n}=$ $\left(I-\omega P_{h_{N}}\right)\left(I-\omega P_{h_{N-1}}\right) \cdots\left(I-\omega P_{h_{1}}\right)\left(u_{H h}-u^{n-1}\right)$, where $I$ denotes the identity operator in $V_{H h}$. Setting $B=\left(I-\omega P_{h_{N}}\right)\left(I-\omega P_{h_{N-1}}\right) \cdots\left(I-\omega P_{h_{1}}\right)$, we obtain that $u_{H h}-u^{n}=B^{n}\left(u_{H h}-u_{H}\right)$.

Proposition 8 If $\omega \in(0 ; 2)$, then the algorithm converges, i.e.

$$
\lim _{n \rightarrow \infty}\left\|u^{n}-u_{H h}\right\|=0 .
$$

In order to prove Proposition 8 let us establish first a more general result.

Let $V$ be a Hilbert space with scalar product $(\cdot, \cdot)$ and denote by $\|\cdot\|$ the induced norm. Consider $V_{1}, V_{2}, \ldots, V_{N}$ closed subspaces of $V$ not reduced to zero. We call $P_{j}: V \rightarrow V_{j} \subset V$ the orthogonal projectors from $V$ onto $V_{j}, j=1,2, \ldots, N$. If $I$ denotes the identity operator in $V$ and $\omega$ is a real parameter, we define the operator $B \in \mathcal{L}(V)$ by

$$
B=\left(I-\omega P_{N}\right)\left(I-\omega P_{N-1}\right) \cdots\left(I-\omega P_{1}\right) .
$$

Introduce the numbers $\gamma_{i j}=\sup _{\substack{v_{i} \in V_{i}, v_{i} \neq 0 \\ v_{j} \in V_{j}, v_{j} \neq 0}} \frac{\left(v_{i}, v_{j}\right)}{\left\|v_{i}\right\|\left\|v_{j}\right\|}=\gamma_{j i} \leq 1$, which are the constants from the corresponding C.B.S. inequalities. Note that $\gamma_{j j}=1$ for $j=$ $1,2, \ldots, N$. Consider the following hypothesis:

Hypothesis $(\overline{\mathbf{H}})$ There exists a constant $\bar{C}_{0}$ such that for all $v \in V$ there exist $v_{j} \in V_{j}, j=1,2, \ldots, N$, satisfying $v=\sum_{j=1}^{N} v_{j}$ and $\sum_{j=1}^{N}\left\|v_{j}\right\|^{2} \leq \bar{C}_{0}^{2}\|v\|^{2}$.

Applying recursively Proposition 3 it is easy to see that $(\overline{\mathrm{H}})$ is satisfied if and only if $V=V_{1}+V_{2}+\ldots+V_{N}$.

Proposition 9 If Hypothesis $(\bar{H})$ is satisfied and if $0<\omega<2$, then the norm of the operator $B$ given by (36) verifies

$$
\|B\| \leq\left(1-\frac{(2-\omega) \omega}{\bar{C}_{0}^{2}(1+\omega \bar{\gamma})^{2}}\right)^{\frac{1}{2}}<1,
$$

where $\bar{\gamma}=\max _{1 \leq j \leq N} \sum_{1 \leq i \leq N, i \neq j} \gamma_{i j}$, with $0 \leq \bar{\gamma} \leq N$. 
Proof The proof can be adapted from [16] in a same way as the one in Proposition 5.

The proof of Proposition 8 now follows easily:

Proof (Proposition 8) This Proposition is proved by applying Proposition 9 to $V=V_{H h}$ and $V_{j}=V_{h_{j}}, j=1,2, \ldots, N$.

Remark that a generalization of Proposition 6 to the case $N>2$ is not straightforward.

\section{Numerical results}

We illustrate the above presented algorithm with the following example: Consider the Poisson-Dirichlet problem

$$
-\Delta u=f
$$

in the domain $\Omega=(-1 ; 1)^{2}, u=0$ on its boundary $\partial \Omega$. Take

$$
f=-4 \eta \chi(R) \frac{R^{2}+R^{4}-\epsilon^{4}}{\left|\epsilon^{2}-R^{2}\right|^{4}} \exp \left(\frac{1}{\epsilon^{2}}\right) \exp \left(\frac{-1}{\left|\epsilon^{2}-R^{2}\right|}\right)
$$

where $R=\sqrt{x_{1}^{2}+x_{2}^{2}}$ and $\chi(R)=1$ if $R \leq \epsilon, \chi(R)=0$ if $R>\epsilon ; \eta$ and $\epsilon$ are parameters. The exact solution to the problem is given by $u=\eta \chi(R) \exp \left(\frac{1}{\epsilon^{2}}\right)$ $\exp \left(\frac{-1}{\left|\epsilon^{2}-R^{2}\right|}\right)$. We choose $\eta=10$ and $\epsilon=0.5$.

Away from the origin $(0,0)$ the solution is smooth. In a region close to $(0,0)$ where the solution has a peak, we need to apply a patch with a finer mesh. For the triangulation of $\bar{\Omega}$, we use a coarse uniform grid with mesh size $H$ and $r=1$. We consider a patch $\Lambda$ with a fine uniform triangulation of size $h$ and $s=1$.

We consider two cases of patches and grids. In a first constellation we take both grids structured. We choose $\Lambda=\left(-\frac{\epsilon}{2}+k h ; \frac{\epsilon}{2}+k h\right)^{2}$ and the mesh sizes $H$ and $h$ such that the fine triangulation is nested in the coarse one for $k=0$. We characterize the grids by $N$ where $4 N$ corresponds to the number of nodes chosen on $\partial \Omega$. Varying the parameter $k$ induces a translation of the patch $\Lambda$ hence leading to situations with non-nested grids. In Figure 3(a) we illustrate the case with $H=1 / 4$, i.e. $N=8, H / h=4$ and $k=2$. A second constellation of interest is where both grids are unstructured and $\Lambda=\left(-\frac{\epsilon}{2} ; \frac{\epsilon}{2}\right)^{2}$. Figure 3(b) illustrates this case with $N=8$ and $H / h=4$.

For numerical quadratures and calculating the errors, we introduce a global fine uniform structured triangulation wherein the fine grid, if structured and $k=0$, is nested. In the nested case, it is an extension of the fine triangulation to the domain $\Omega$, taken two times finer in order to minimize the projection errors introduced when comparing the results. We use the software Freefem++ [24] to generate the grids and implement the algorithm. 


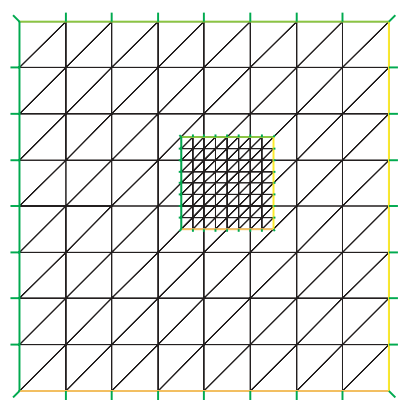

(a) Non-nested structured grids with parameter $k=2$.

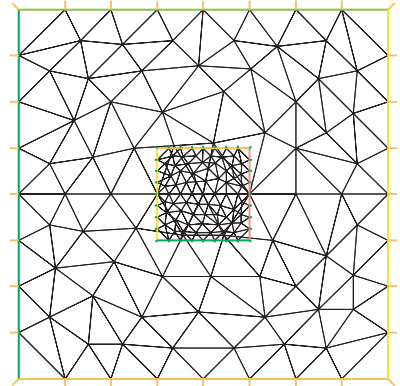

(b) Unstructured grids.

Fig. 3 Illustrations with $N=8$ and $H / h=4$ of some patches and grids considered

\subsection{Numerical evaluation of $\tilde{\gamma}$}

As mentioned at the end of Section 4 we can evaluate the parameter $\tilde{\gamma}$ of two spaces $V_{H}$ and $V_{h}$ by running Algorithm 1, estimating the spectral radius $\rho$ of the iteration operator $B$ and using the result of Proposition 6 linking $\rho$ to $\tilde{\gamma}$ for a given $\omega$. Recall that, if $\omega=1$ we have $\tilde{\gamma}=\sqrt{\rho(B)}$, and that $\rho(B)$ is the absolute value of the largest eigenvalue. A convenient numerical evaluation of the spectral radius is done by setting $f=0$, starting in practice with any initial condition $u^{0}$ non-zero, and evaluating $\rho(B)$ as $\sqrt[n]{\left\|u^{n}\right\|}$ for large $n$. Indeed, we can prove that

$$
\lim _{n \rightarrow \infty} \sqrt[n]{\left\|u^{n}\right\|}=\lim _{n \rightarrow \infty} \sqrt[n]{\left\|B^{n} u^{0}\right\|}=\rho
$$

if $u^{0}$ has a non-zero component in the direction of the eigenvector(s) corresponding to the eigenvalue(s) giving the spectral radius. Note that we do not use the standard power method as it does not apply when $\rho(B)$ corresponds to a complex eigenvalue.

For the case of right isosceles triangles as presented in Figure 2 we find the estimates of Table 2 for $\gamma=\tilde{\gamma}$. These results are to be compared with the estimated upper bounds presented earlier in Table 1: we remark that they are not sharp. Note that due to the relation (14) we also have an estimate for the optimal constant $C_{0}$ of (13).

Before presenting the estimates for $\tilde{\gamma}$ in the cases of the above introduced grids, it is worth illustrating the fitting of formula (29) for $\rho$ with the numerical estimates. In the case of nested grids with $N=8, H / h=4$ we obtain $\tilde{\gamma}=0.306$ for $\omega=1$. In Figure 4 we plot $\rho(\tilde{\gamma}=0.306, \omega)$ and the corresponding numerical results for $\rho$ using (37) for $\omega \in(0 ; 2)$.

In order to get an idea of the convergence behavior of the algorithm for the different grid structures presented at the beginning of this Section, it is interesting to evaluate $\tilde{\gamma}$. The convergence factor of the algorithm being bounded by $\|B\|$ (see Prop. 2), with $\|B\|=\tilde{\gamma}$ for $\omega=1, \tilde{\gamma}$ is a bound for the convergence factor. In Table 3 we present estimates for $\tilde{\gamma}$ in the case of structured and unstructured 


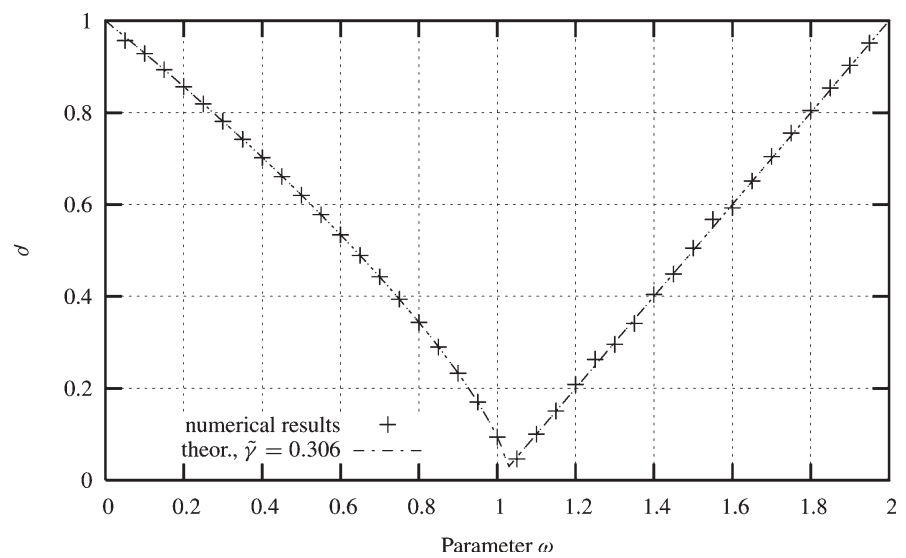

Fig. 4 Comparison of numerical estimates and theoretical results for $\rho$ for different parameter $\omega$ in the case of nested grids, $N=8$ and $H / h=4$

grids. The parameter $\tilde{\gamma}$ is given in the case of structured nested grids $(k=0)$, first for fixed $N=8$ and different ratios $H / h$ (Table 4(a)), then for fixed $H / h=4$ and variable $N$ (Table 4(b)). Table 4(c) gives estimates for $\tilde{\gamma}$ for some non-nested constellations with structured grids in the case $N=8$ and $H / h=4$. Finally we give some estimates in the case with unstructured grids for variable ratios $H / h$ (Table 4(d)).

\subsection{Convergence of the algorithm}

The objective of this paragraph is to illustrate the convergence of the algorithm on the chosen example. In the sequel we consider $\omega=1$. We introduce the stopping criterion $\left\|u^{n}-u^{n-1}\right\| /\left\|u^{n}\right\|<\epsilon_{1}$ where $n, n=1,2, \ldots$, is the iteration number. If this criterion yields true at iteration $n_{c}$, we define $u_{H h}=u^{n_{c}}$. To verify that the algorithm has well converged, we check that $u_{H h}$ satisfies a second criterion, namely $\left\|\bar{u}_{H h}-u_{H h}\right\| /\left\|\bar{u}_{H h}\right\|<\epsilon_{2}$, where $\bar{u}_{H h}=u^{n_{c}+p}, p=20$. We choose $\epsilon_{1}=10^{-4}$ and $\epsilon_{2}=10 \epsilon_{1}$. We define the relative error at iteration $n, n=0,1, \ldots, n_{c}$, by $e^{n}=\left\|\bar{u}_{H h}-u^{n}\right\| /\left\|\bar{u}_{H h}\right\|$. The evolution of this error through the iterative process gives information about the speed of convergence of the algorithm.

The type of grids we use is an important element for the convergence rate of the algorithm. This can be readily seen through the $\tilde{\gamma}$-estimates presented in Table 3 . The curves of Figure 5 illustrate the influence of the chosen grids. We compare the behavior for the cases of nested $(k=0$ and $k=4)$, non-nested $(k=2)$ and unstructured grids with $N=8$ and $H / h=4$. In the nested case, the algorithm converges very fast, in only a couple of steps. The results correspond to the behavior of the estimated bound $\tilde{\gamma}$ for the convergence factor. The rate of convergence can be well foreseen by the estimate of $\tilde{\gamma}$. From Table 3 we have that $\tilde{\gamma}=0.306$ resp. 0.474 in the nested cases with $k=0$ and $4, \tilde{\gamma}=0.785$ in the considered non-nested case and $\tilde{\gamma}=0.944$ in the case of unstructured and completely uncorrelated grids. Note that even in the latter most general case the algorithm converges steadily. For the 


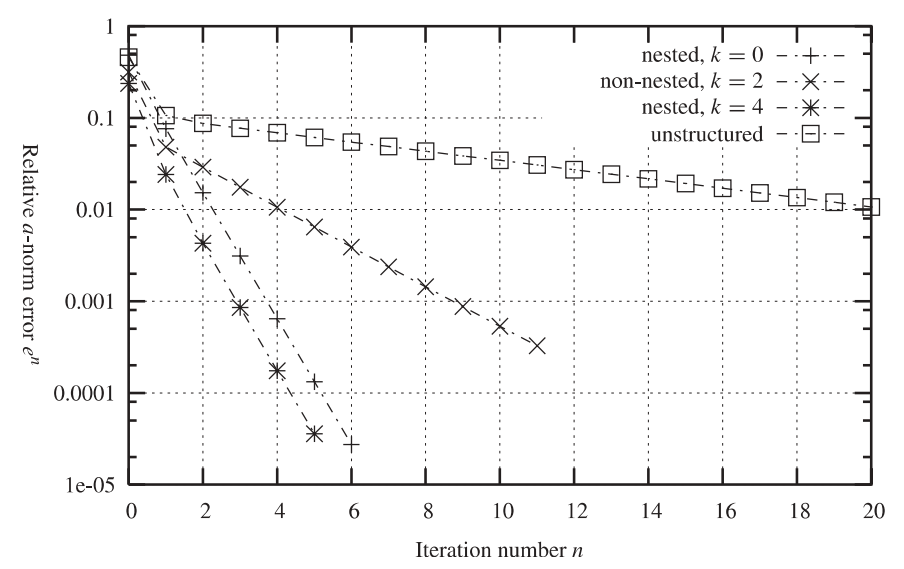

Fig. 5 Convergence of the algorithm for different cases of patches and grids

readibility of the graphic, we show only the evolution of the error through the first 20 iterations.

Similarly, the convergence behavior of the algorithm follows the convergence rate bound $\tilde{\gamma}$ (Table 3 ) in the cases with fixed $N$ and variable $H / h$, respectively with fixed $H / h$ and variable $N$. The convergence is slightly slower with increasing $H / h$ for fixed $N$. The same holds for increasing $N$ and fixed $H / h$.

\subsection{Convergence in the grid size}

Up to now, we have only considered illustrations for the convergence of the algorithm, i.e. obtaining the approximation $u_{H h}$ to the exact solution $u$. To assess the convergence of $u_{H h}$ in $H$ and $h$ given by the a priori estimate of Proposition 1, we introduce the relative error $e_{H h}=\left\|u-u_{H h}\right\| /\|u\|$. This error is evaluated by interpolating $u$ and $u_{H h}$ on the fine uniform structured grid. The result $u_{H h}$ is obtained here by requiring $a$-norm convergence of the algorithm. On the graphics of Figure 6 we show the relative $L^{2}-, H^{1}$ - and $L^{\infty}$-norm errors $e_{H h}$ for increasing $N, N=8,16,32,64$, with $H / h=2$ fixed, in the case of nested $(k=0)$ and unstructured grids. In both cases, we observe optimal convergence in the mesh size $H=2 / N$ : we observe $h^{2}$-accuracy for the $L^{2}$-norm and rate of convergence one for the $H^{1}$-norm.

\section{Concluding remarks}

We have presented and analyzed a method for solving numerically problems with multi-scale data. The method uses patches whose triangulations are not necessarily nested. In Section 6 we have only presented results in the two-level case. Remark that the implementation of the generalization to multiple levels, as given in 


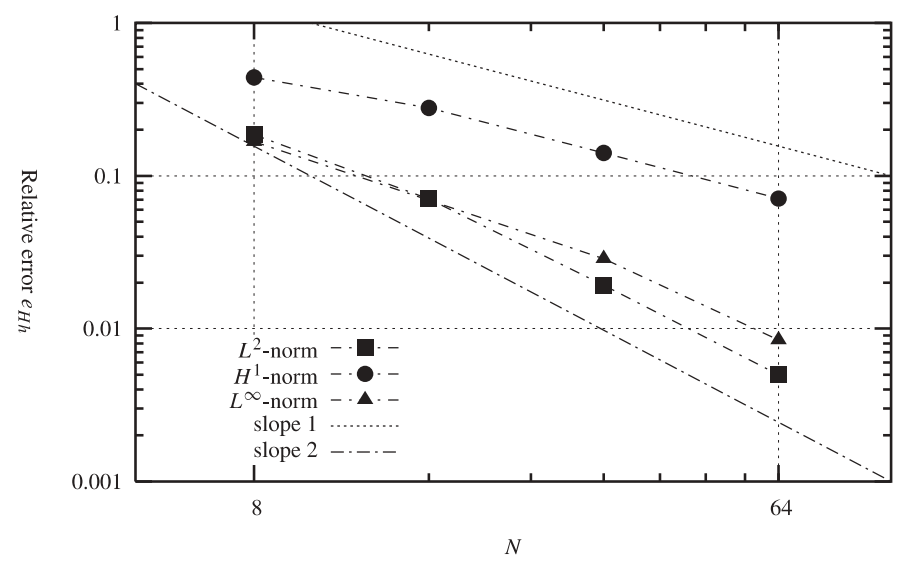

(a) Nested grids.

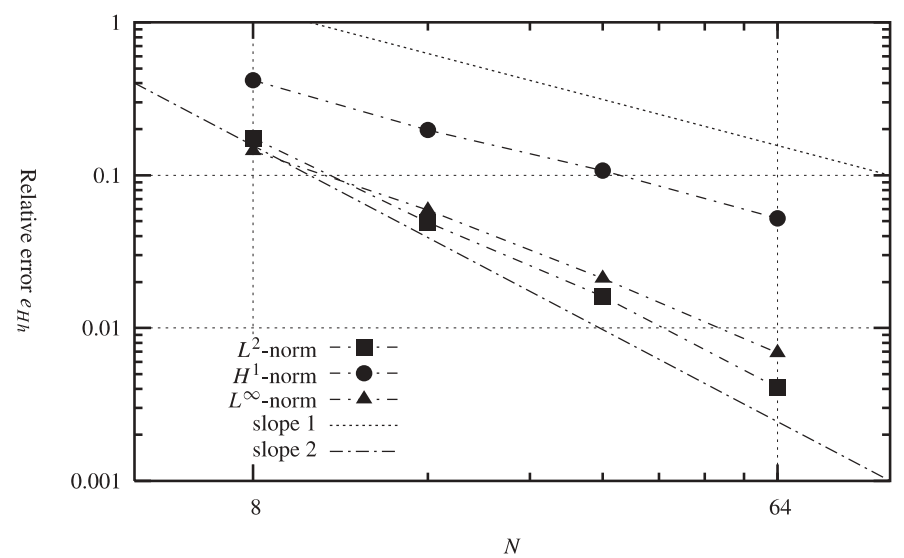

(b) Unstructured grids.

Fig. 6 Convergence in the mesh size with $H / h=2$

Section 5, leads to efficient programs, in particular if a telescopic set of well-adapted patches is chosen. At each level of refinement the correction in all non-overlapping patches can be parallelized. Using patches to connect problems in adjacent domains with nonconforming grids leads to efficient coupling techniques. Large domains can be split into non-overlapping subdomains for parallel treatment and patches are to be used at a second level to connect the subdomains. Applications of the method include all problems where local refinement is necessary, as, for example, in boundary layer problems. We are looking forward to presenting results illustrating different types of implementations and applications of the algorithm. 


\section{References}

1. Achchab, B., Maître, J.-F.: Estimate of the constant in two strengthened C.B.S. inequalities for F.E.M. systems of 2D elasticiy: Application of multilevel methods and a posteriori error estimators. Numer. Linear Algebra Appl. 3(2), 147-159 (1996)

2. Achdou, Y., Maday, Y.: The mortar element method with overlapping subdomains. SIAM J. Numer. Anal. 40(2), 601-628 (2002)

3. Adams, R.A., Fournier, J.J.F.: Sobolev Spaces. Vol. 140 Pure and Applied Mathematics. Elsevier Science, Oxford, 2nd edition, 2003

4. Axelsson, O.: On multigrid methods of the two-level type. In: W. Hackbusch, U. Trottenberg (eds.), Multigrid Methods. Vol. 960 of Lecture Notes in Mathematics. Springer-Verlag, Berlin, 1981 pp. 352-367

5. Axelsson, O.: Stabilization of algebraic multilevel iteration methods; additive methods. $\mathrm{Nu}-$ mer. Algorithms 21, 23-47 (1999)

6. Axelsson, O., Blaheta, R.: Two simple derivations of universal bounds for the C.B.S. inequality constant. Report No. 0133, Dept. of Mathematics, University of Nijmegen, The Netherlands, 2001

7. Axelsson, O., Gustafsson, I.: Preconditioning and Two-Level Multigrid methods of arbitrary degree of approximation. Math. Comp. 40(161), 219-242 (1983)

8. Axelsson, O., Vassilevski, P.S.: Algebraic multilevel preconditioning methods. I. Numer. Math. 56, 157-177 (1989)

9. Axelsson, O., Vassilevski, P.S.: Algebraic multilevel preconditioning methods. II. SIAM J. Numer. Anal. 27(6), 1569-1590 (1990)

10. Bank, R.E., Dupont, T.F., Yserentant, H.: The hierarchical basis multigrid method. Numer. Math. 52, 427-458 (1988)

11. Bank, R.E., Smith, R.K.: A posteriori error estimates based on hierarchical bases. SIAM J. Numer. Anal.30(4), 921-935 (1993)

12. Blaheta, R.: Space decomposition preconditioners and parallel solvers. In Conference Proceedings ENUMATH 2003, Prague. Springer-Verlag, to appear

13. Braess, D.: The Contraction Number of a Multigrid Method for Solving the Poisson Equation. Numer. Math. 37, 387-404 (1981)

14. Braess, D.: The Convergence Rate of a Multigrid Method with Gauss-Seidel Relaxation for the Poisson Equation. In: W. Hackbusch, U. Trottenberg(eds.), Multigrid Methods. Vol. 960 Lecture Notes in Mathematics, Springer-Verlag, Berlin, 1981 pp. 368-386

15. Bramble, J.H., Ewing, R.E., Pasciak, J.E., Schatz, A.H.: A preconditioning technique for the efficient solution of problems with local grid refinement. Comput. Methods Appl. Mech. Engrg. 67, 149-159 (1988)

16. Bramble, J.H., Pasciak, J.E., Wang, J., Xu, J.: Convergence estimates for product iterative methods with applications to domain decomposition. Math. Comp. 57(195), 1-21 (1991)

17. Brezzi, F., Lions, J.-L., Pironneau, O.: Analysis of a Chimera method. C. R. Acad. Sci. Paris Ser. I 332, 655-660 (2003)

18. Cai, X.-C., Widlund, O.B.: Multiplicative Schwarz algorithms for some nonsymmetric and indefinite problems. SIAM J. Numer. Anal. 30(4), 936-952 (1993)

19. Chan, T.F., Smith, B.F., Zou, J.: Overlapping Schwarz methods on unstructured meshes using non-matching coarse grids. Numer. Math. 73, 149-167 (1996)

20. Ciarlet, Ph.G.: Numerical Analysis of the Finite Element Method. Séminaire de Matheématiques Supérieures. Les Presses de L'Université de Montréal, Canada, 1976

21. Eijkhout, V., Vassilevski, P.: The role of the strengthened Cauchy-Buniakowskii-Schwarz inequality in multilevel methods. SIAM Rev. 33(3), 405-419 (1991)

22. Glowinski, R., He, J., Rappaz, J., Wagner, J.: Approximation of multi-scale elliptic problems using patches of finite elements. C. R. Acad. Sci. Paris Ser. I 337, 679-684 (2003)

23. Griebel, M., Oswald, P.: On the abstract theory of additive and multiplicative Schwarz algorithms. Numer. Math. 70, 163-180 (1995)

24. Hecht, F., Pironneau, O., Ohtsuka, K.: Freefem++ ver. 1.28. http://www.freefem.org

25. Jung, M., Maître, J.-F.: Some Remarks on the Constant in the Strengthened CBS Inequality: Estimate for hierarchical finite element discretizations of elasticity problems. Numer. Methods Partial Differential Equations 15(4), 469-487 (1999)

26. Laydi, M.R.: Convergence d'un schéma itératif explicite. C. R. Acad. Sci. Paris Ser. I 326, 511-514 (1998) 
27. Berlin Maître, J.-F., Musy, F.: The contraction number of a class of two-level methods; an exact evaluation for some finite element subspaces and model problems. In: W. Hackbusch, U. Trottenberg (eds.), Multigrid Methods. Vol. 960 of Lecture Notes in Mathematics. Springer-Verlag, 1981, pp. 535-544

28. Margenov, S.D.: Upper bound of the constant in the strengthened C.B.S. inequality for FEM 2D elasticity equations. Numer. Linear Algebra Appl. 1(1) 65-74 (1994)

29. McCormick, S.F.: Fast Adaptive Composite grid (FAC) Methods: Theory for the variational Case. In: K. B \{ohner, H.J. Stetter (eds.), Defect Correction Methods. Theory and Applications. Vol.5 of Computing Supplementum, Springer 1984, pp. 115-121

30. McCormick, S.F., Ruge, J.W.: Unigrid for multigrid simulation. Math. Comp. 41(163), 43-62 (1983)

31. McCormick, S.F., Thomas, J.: The Fast Adaptive Composite Grid (FAC) Method for Elliptic Equations. Math. Comp. 46(174), 439-456 (1986)

32. Quarteroni, A., Valli, A.: Domain Decomposition Methods for Partial Differential Equations. Numerical Mathematics and Scientific Computation. Oxford Science Publications, Oxford, 1999

33. Schwarz, H.A.: Ueber einen Grenzübergang durch alternirendes Verfahren. Vol. 2 of Gesammelte Mathematische Abhandlungen. Chelsea Publishing Company, Bronx, New York, 1972, pp. 133-143

34. Smith, B., Bjørstad, P., Gropp, W.: Domain Decomposition: Parallel Multilevel Methods for Elliptic Partial Differential Equations. Cambridge University Press, Cambridge, 1996

35. Steger, J.L., Benek, J.A.: On the use of composite grid schemes in computational aerodynamics. Comput. Methods Appl. Mech. Engrg. 64, 301-320 (1987)

36. Wang, J.: Convergence analysis of the Schwarz algorithm and multilevel decomposition iterative methods II: Nonselfadjoint and indefinite elliptic problems. SIAM J. Numer. Anal. 30(4), 953-970 (1993)

37. Widlund, O.B.: Domain decomposition methods for elliptic partial differential equations. In: H. Bulgak, C. Zenger (eds.), Error control and adaptativity in scientific computing. Kluwer Academic Publishers, 1999, pp. 325-354

38. Wohlmuth, B.I.: Discretization methods and iterative solvers based on domain decomposition. Vol. 17 of Lecture Notes in Computational Science and Engineering. Springer, 1991

39. Xu, J.: Iterative methods by space decomposition and subspace correction. SIAM Rev. 34(4), 581-613 (1992)

40. Xu, J.: An introduction to multilevel methods. In: M. Ainsworth, J. Levesley, W.A. Light, M. Marletta (eds.), Wavelets, multilevel methods and elliptic PDEs, Numerical Mathematics and Scientific Computation. Oxford University Press, 1997, pp. 213-302

41. Xu, J.: The method of subspace corrections. J. Comput. Appl. Math. 128, 335-362 (2001)

42. Xu, J., Zikatanov, L.: The method of alternating projections and the method of subspace corrections in Hilbert space. PennState, Department of Mathematics, AM223, 2000

43. Yosida, K.: Functional Analysis. Vol. 123 of A series of Comprehensive Studies in Mathematics. Springer-Verlag, Berlin, 6th edititon, 1980

44. Yserentant, H.: On the multi-level splitting of finite element spaces. Numer. Math. 49, 379$412(1986)$

45. Yserentant, H.: Hierarchical Bases. In: R.E. O'Malley (ed), Proceedings of the Second International Conference on Industrial and Applied Mathematics. SIAM, 1991, pp. 256-276

46. Yserentant, H.: Old and new convergence proofs for multigrid methods. Acta Numer. 285$326(1993)$ 